\title{
The Diagnostic Accuracy of in vivo Confocal Scanning Laser Microscopy Compared to Dermoscopy of Benign and Malignant Melanocytic Lesions: A Prospective Study
}

\author{
Richard G.B. Langley ${ }^{a}$ Noreen Walsh ${ }^{c}$ Ashley E. Sutherland ${ }^{a} \quad$ Iva Propperova ${ }^{a}$ \\ Laura Delaney $^{\mathrm{a}}$ Steven F. Morris ${ }^{\mathrm{b}}$ Christopher Gallant ${ }^{\mathrm{a}}$ \\ ${ }^{a}$ Division of Dermatology, Department of Medicine, ${ }^{b}$ Division of Plastic and Reconstructive Surgery, \\ Department of Surgery, and 'Department of Pathology, Dalhousie University, Halifax, N.S., Canada
}

\section{Key Words}

Confocal scanning laser microscopy, diagnostic accuracy •

Melanoma $\cdot$ Dermoscopy

\begin{abstract}
Background: The diagnosis of melanoma at an early, curable stage is an important challenge for clinicians. Confocal scanning laser microscopy (CSLM) is a high-resolution, noninvasive technology that may facilitate improved diagnostic accuracy over clinical examination. The aim of this study was to evaluate the diagnostic accuracy of CSLM compared to dermoscopy in a prospective examination of benign and malignant melanocytic lesions. Methods: 125 patients with suspicious pigmented lesions were prospectively recruited to undergo a clinical, dermoscopic and CSLM examination. A diagnosis was made preoperatively with each technique, and the lesion was then excised and diagnosed using histopathology. Results: 125 patients with 125 lesions were studied comprising 88 melanocytic nevi and 37 melanomas. Dermoscopy had a sensitivity of $89.2 \%$, a specificity of $84.1 \%$, a positive predictive value of $70.2 \%$ and a negative predictive value of $94.9 \%$. CSLM was found to have a sensitivity of
\end{abstract}

$97.3 \%$, a specificity of $83.0 \%$, a positive predictive value of $70.6 \%$ and a negative predictive value of $98.6 \%$. No melanomas were misidentified when both techniques were used together. Conclusions: CSLM had a relatively higher sensitivity than dermoscopy; however, the specificity was similar with CSLM and dermoscopy. These results suggest that dermoscopy and CSLM are complementary.

Copyright $\odot 2007$ S. Karger AG, Basel

\section{Introduction}

The diagnosis of primary cutaneous melanoma at an early, curable stage is an important challenge facing clinicians. The diagnostic accuracy of clinical examination is in the order of $64-80 \%$ [1-3], however, prompting the development of noninvasive technologies such as dermoscopy [4-7], image analysis [8] and more recently in vivo reflectance mode confocal scanning laser microscopy (CSLM) [9-22]. In addition, improved diagnostic accuracy could help reduce the unnecessary removal of benign lesions with the associated morbidity.

\section{KARGER}

Fax +41613061234 E-Mail karger@karger.ch www.karger.com
(C) 2007 S. Karger AG, Basel

$1018-8665 / 07 / 2154-0365 \$ 23.50 / 0$

Accessible online at:

www.karger.com/drm
Richard G.B. Langley, MD, FRCPC

Centre for Clinical Research Building, Room 121, QEII Health Sciences Centre

Division of Dermatology, Department of Medicine, Dalhousie University 5790 University Avenue, Halifax, NS B3H 1V7 (Canada)

Tel. +1 902425 8038, Fax +1 902421 1580, E-Mail richard.langley@dal.ca 
Dermoscopy is a noninvasive technique which has currently received the widest clinical use, and there is evidence that it can improve the diagnostic accuracy in the assessment of benign and malignant melanocytic lesions [4-7, 23-25]. CSLM is a promising noninvasive imaging technique which provides high-resolution, instantaneous, cellular-level detail of human skin in vivo. The morphological features of benign and malignant melanocytic lesions have been described with CSLM [11-22], and more recently the sensitivity and specificity of these features and this method have been examined in a retrospective analysis, facilitating an improved diagnostic accuracy of melanoma over clinical examination alone [21]. A prospective study comparing the diagnostic accuracy of CSLM compared to dermoscopy has not been reported to date.

The aim of this study was to systematically evaluate the diagnostic accuracy of in vivo reflectance CSLM compared to dermoscopy in a prospective series of benign and malignant melanocytic lesions.

\section{Materials and Methods}

\section{Patients}

One hundred twenty-seven patients were recruited prospectively during the period from February 2002 to May 2005 from the Division of Dermatology Pigmented Lesion Clinic and the Plastic Surgery Clinics at the Queen Elizabeth II Health Sciences Centre, Dalhousie University, for a study approved by the Research Ethics Board. Two patients were excluded from the database due to technical difficulties with the imaging. One hundred twenty-five patients were enrolled into the final study database. All patients gave both oral and written consent prior to the start of any study-related procedures. Both male and female patients aged 16 years and older were recruited for the study. All recruited patients were scheduled for biopsy of their lesions due to clinical suspicion of malignancy determined by clinical appearance or a history of change in the lesion. Patients were also asked to provide a brief clinical history before imaging. Patients were excluded from the study if their lesions were not amenable to CSLM (i.e. physically inaccessible site), or if they had a previous diagnostic biopsy done on the lesion.

\section{Imaging}

Clinical, dermoscopic and confocal examinations were conducted sequentially by a single reviewer (R.L.). The lesion in question was initially examined clinically and a single diagnosis recorded. Next, dermoscopy was performed and a diagnosis rendered using the pattern analysis method. A clinical photograph was obtained with a Nikon D1X digital camera, and with a Nikon F401s camera with a 60-mm lens with dermatophot attachment. The lesion as well as adjacent, uninvolved, clinically normal, control skin were then imaged with a confocal scanning laser microscope (Vivascope 1000, Lucid Inc., Henrietta, N.Y., USA), and following completion of imaging a diagnosis was recorded. The full technical details of the CSLM have previously been described $[9,10]$. Briefly, for confocal imaging, a drop of oil was applied to the control/lesional skin, followed by a metal adaptor ring with a tape adhesive. The adaptor ring was partially filled with sterile water and the CSLM lens was then attached to the adaptor ring. The confocal scanning laser microscope was scanned with a field of view of $450 \times 400 \mu \mathrm{m}$ which was scanned repeatedly over a total area of $13 \mathrm{~mm}$. A standardized CSLM evaluation was performed with imaging in a stepwise and sequential manner. CSLM was initially performed stepwise through all layers of the epidermis through to the papillary dermis, followed by the sequential repositioning of the objective lens to adjacent areas which provided visualization of the entire lesion in a grid-like manner giving a 3-dimensional view of the lesional/control skin.

A single observer with experience in CSLM performed the imaging and examined all images in real-time (R.L.). The cellular and morphological details of each lesion were analyzed using the CSLM images obtained. During analysis of the confocal images, architectural and cytological features were carefully examined, and a diagnosis was made after the completion of imaging based on these features. The morphological criteria that were used to establish a confocal diagnosis of melanoma and nevi were based on the criteria we described in our initial series [12]. For the diagnosis of melanoma, the architectural and cytological features included: epidermal disarray with loss of the normal honeycomb pattern; a grainy image; pagetoid cells in the epidermis; complex branching dendrites or dendritic cells; atypical and pleomorphic refractile cells, and the presence of bright, highly refractile particles. For the diagnosis of nevi, the architectural and cytological features included: a normal epidermal architecture with a regular honeycombed pattern; the presence of junctional or dermal nests, and monomorphic refractile cells. For benign melanocytic lesions, it was expected that dendrites, if present, would be rare and not have complex branching patterns.

During confocal imaging, digital still images were captured and stored with dedicated software (Vivascope 1000). The images were then stored on an IBM Netvista computer and separately on compact disks.

When CSLM imaging was complete, the lesions were removed by excisional biopsy. A definitive diagnosis was made by a dermatopathologist (N.W.) with conventional hematoxylin-eosinstained histopathological sections. The diagnoses made from CSLM analysis were then compared to the diagnoses made using dermoscopy and pathology reports.

Statistical Analysis

Statistical analysis was performed using the program SAS (version 9.1, 2002). To perform statistical analysis on the data, each diagnosis was converted into a numerical value where benign nevi $=1$ and melanoma $=2$. Statistical analyses (sensitivity, specificity, positive predictive value and negative predictive value) were performed using the diagnoses made by comparing dermoscopic and CSLM diagnoses with the histopathological diagnoses. The sensitivity of a diagnostic technique determines the probability of a positive test result in a person who has the disease. This is defined according to the equation: $\mathrm{TP} /(\mathrm{TP}+\mathrm{FN})$ [26]. In this equation, TP is the number of true-positive and FN that of false-negative results. The specificity of a diagnostic 

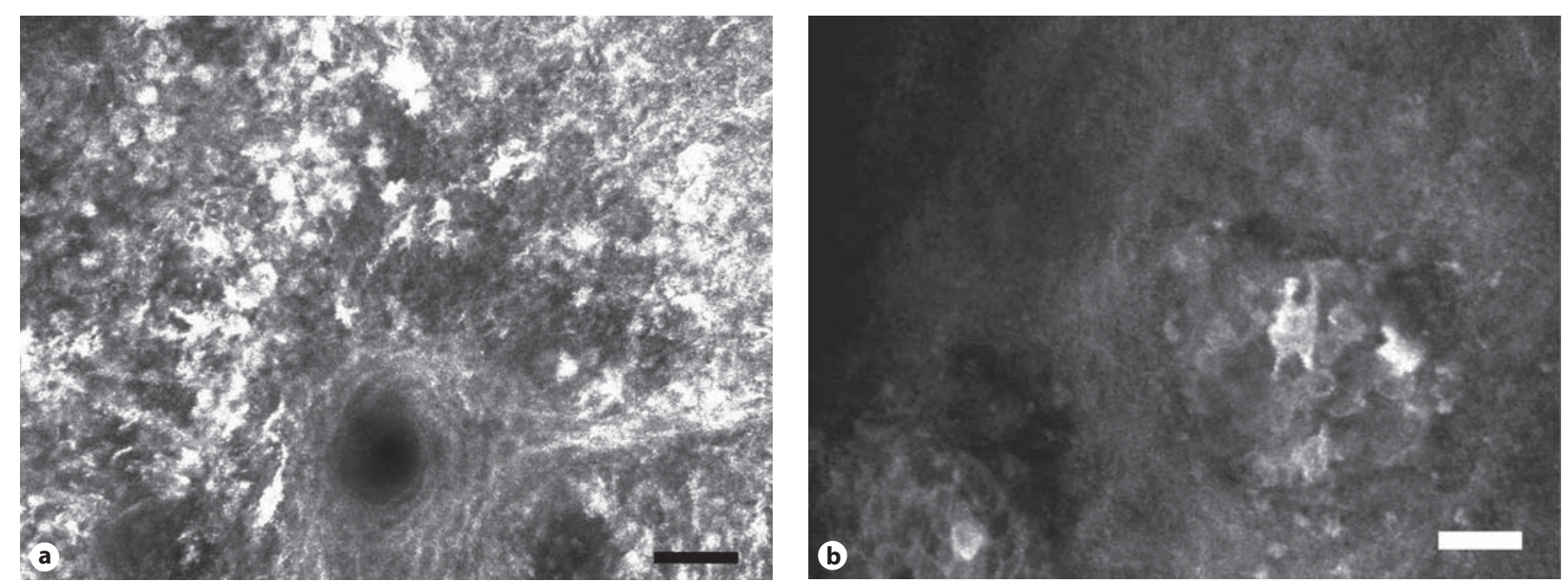

Fig. 1. a Melanoma in situ, from the cheek of a 46-year-old male. Pagetoid proliferation of multiple highly refractile pleomorphic atypical cells with prominent dendrites adjacent to a hair follicle (scale bar $=50 \mu \mathrm{m}$ ). b Melanoma $1.2 \mathrm{~mm}$, Clark's level III, from the right upper back of a 37-year-old female. CSLM reveals atypical, bright, dendritic cells in the dermis, with polymorphous atypical refractile cells. Scale bar $=50 \mu \mathrm{m}$.

technique refers to the probability of a negative test result in a person who does not have the disease according to the equation: $\mathrm{TN} /(\mathrm{TN}+\mathrm{FP})[26]$. In this equation, $\mathrm{TN}$ is the number of truenegative and FP that of false-positive results. The predictive value of a diagnostic test is also important in determining the applicability of the diagnostic technique. The positive predictive value is defined by the equation $\mathrm{TP} /(\mathrm{TP}+\mathrm{FP})$ and is the probability that a patient has the condition given a positive test result. The negative predictive value is determined by the equation TN/ $(\mathrm{TN}+\mathrm{FN})$ and is the probability that a patient does not have the condition given a negative test result [26]. The histopathology results were taken as the definitive diagnosis for each lesion examined.

Each diagnostic tool was compared to the others in an attempt to determine the best diagnostic tool or best combination of diagnostic tools. An overall extended McNemar test of significance for sensitivity and specificity combined was performed [27]. The McNemar test is a $\chi^{2}$ test with 1 degree of freedom. Separately, sensitivity and specificity for the two diagnostic tools were compared. The difference and corresponding confidence interval for each outcome were then calculated.

A measure of test agreement using the $\kappa$ statistic for interrater reliability was also performed as a secondary method of comparing the two diagnostic tests. The following values of $\kappa$ help define the reliability of a test: a $\kappa$ value of 1 indicates perfect agreement, whereas $\kappa$ values falling above 0.8 are considered to have excellent agreement; $\kappa$ values which lie between 0.6 and 0.8 are considered to have substantial agreement, while values falling between 0.4 and 0.6 indicate moderate agreement [26].

A Student's t test was performed on the demographic information collected from the patients to determine any statistical significance within the study group.

Diagnostic Accuracy of CSLM Compared to Dermoscopy in Melanocytic Lesions

\section{Results}

In total, 125 patients were eligible for enrollment into the study which included 125 lesions and 30,089 CSLM images. Representative confocal images are provided in figure 1 . These cases included 88 melanocytic nevi and 37 melanomas including 18,045 images of nevi and 12,044 CSLM images of melanoma. All lesions were verified by histopathology. Histopathological review established that $40.5 \%(15 / 37)$ of the melanomas were diagnosed as in situ, while $59.5 \%(22 / 37)$ melanomas were invasive. The median Breslow thickness for the invasive melanomas was $0.62 \mathrm{~mm}(0.20-7.92 \mathrm{~mm})$. The subjects ranged in age from 16 to 84 years old with a mean age of 44.2 years. The average age for males was 48.2 years (standard deviation, $\mathrm{SD}, 16.2$ ) and the average age for females was 40.2 (SD 15.0; $\mathrm{p}=0.0054)$. The mean age for those diagnosed as having melanoma was 51.5 years (SD 14.5). The mean age of patients with a diagnosis of benign nevi was 35.8 years (SD 13.7; $\mathrm{p}<0.0001$ ). It was found that females enrolled in this study were significantly younger than males ( $\mathrm{p}=0.0054)$, and those diagnosed as having melanoma were significantly older than those with benign nevi $(\mathrm{p}<$ 0.0001).

The diagnostic accuracies of dermoscopy and CSLM were analyzed. The sensitivity and specificity of dermoscopy for the diagnosis of melanoma were 89.2 and $84.1 \%$, respectively. The sensitivity and specificity of CSLM for 
Table 1. The specificity, sensitivity, positive and negative predictive values for dermoscopic and CSLM diagnoses for malignant melanoma and benign nevi

\begin{tabular}{llllll}
\hline $\begin{array}{l}\text { Diagnostic } \\
\text { test }\end{array}$ & $\begin{array}{l}\text { Number of benign lesions } \\
\text { correctly diagnosed } \\
(88 \text { total) }\end{array}$ & $\begin{array}{l}\text { Number of malignant mela- } \\
\text { nomas correctly diagnosed } \\
(37 \text { total })\end{array}$ & $\begin{array}{l}\text { Specificity } \\
\%\end{array}$ & $\begin{array}{l}\text { Sensitivity } \\
\%\end{array}$ & $\begin{array}{l}\text { Positive } \\
\text { predictive } \\
\text { value, \% }\end{array}$ \\
\hline Dermoscopy & 74 & 33 & 84.1 & $\begin{array}{l}\text { Negative } \\
\text { predictive } \\
\text { value, } \%\end{array}$ \\
CSLM & 73 & 36 & 83.0 & 97.2 & 70.2 \\
\hline
\end{tabular}

the diagnosis of melanoma were 97.3 and $83.0 \%$, respectively. Sensitivities, specificities, positive and negative predictive values for dermoscopic and CSLM diagnosis can be found in table 1 .

The overall McNemar test of significance for specificity and sensitivity combined was rejected at the $5 \%$ significance level $(\mathrm{p}=0.3932)$. Therefore, no significant difference was found between the sensitivities or specificities of the two methods. CSLM had a higher sensitivity compared to dermoscopy. The difference was $8.11 \%$ (confidence interval -3.15 to $19.35 \%)$. The McNemar test statistic was not statistically significant $(p=0.1797)$. Dermoscopy had a higher specificity with a difference of $1.14 \%$ (confidence interval -7.39 to $9.67 \%$ ). The McNemar test statistic for specificity was not statistically significant $(\mathrm{p}=0.7963)$.

It was found using the $\kappa$ statistic for interrater reliability that CSLM and pathology had the highest $\kappa$ value of 0.72 indicating substantial agreement between the two diagnostic methods. The use of dermoscopy and CSLM produced a $\kappa$ value of 0.67 indicating substantial agreement using the two diagnostic techniques.

Diagnoses made using dermoscopy and CSLM techniques together agreed on 73 out of 88 total benign nevi, and on 32 out of 37 malignant melanomas. In our results, there were 5 melanomas for which CSLM and dermoscopy produced differing diagnoses. In these cases, CSLM correctly classified 4 of the melanomas, whereas dermoscopy correctly classified the other melanoma. There were no cases where melanoma was misdiagnosed when CSLM and dermoscopy were used together. There were 15 benign nevi for which the diagnoses made by dermoscopy and CSLM differed. Of these, dermoscopy provided the correct diagnosis 9 times, and CSLM made the correct diagnosis 6 times. There were 7 benign nevi for which both diagnoses were incorrect.

Two of the melanomas were misdiagnosed by the investigator using dermoscopy, but correctly diagnosed by CSLM were amelanotic/hypomelanotic melanomas.

\section{Discussion}

CSLM is a promising noninvasive imaging technique that may facilitate the diagnosis of cutaneous melanoma and the discrimination of benign melanocytic lesions. We reported the initial series of benign and malignant melanocytic lesions using a CSLM prototype that was designed and constructed in the Wellman Laboratories of Massachusetts General Hospital [11, 12]. In our investigations, we examined 40 pigmented lesions involving 34 nevi and 6 melanomas [12]. Melanin provided a natural contrast agent in CSLM enabling high-resolution imaging. Melanocytic nevi were characterized by the presence of bright, refractile, monomorphic cells with dark nuclei, and were clustered into dense nests. The typical features of melanoma using CSLM included architectural disarray of the stratum spinosum and an inability to detect keratinocyte cell borders (focally or diffuse). In addition, cytological atypia was recognized with complex dendritic structures evident within the epidermis. These qualitative findings have been confirmed, extended and quantified in several other series using commercially available CSLM [14-21]. These studies have helped establish the key in vivo reflectance confocal microscopic morphological features of benign and malignant pigmented skin lesions, and a consensus statement of standard terminology for CSLM has recently been completed [28].

This work has helped define and standardize the key morphological features of CSLM, however, the diagnostic accuracy of this technique is still being determined. A recent retrospective study by Gerger et al. [21] examined the sensitivity and specificity of CSLM. In this study, 88 patients were imaged with CSLM (Vivascope 1000, Lucid Inc., Rochester, N.Y., USA) including 117 melanocytic lesions comprising 90 nevi (30 histologically verified) and 27 melanomas (all histologically verified). In this study, 5 independent observers (2 residents, 1 senior physician, 2 dermatopathologists), with no prior experience with 
CSLM, received a 30-min oral presentation involving standardized instruction about the diagnostic features of melanocytic lesions imaged by CSLM. The independent observers then examined the diagnostic images shown on a computer screen and evaluated the lesion as being a benign nevus or melanoma. The observers were blinded to the clinical, dermoscopic or histopathological diagnoses, and no clinical diagnosis of surface microscopy was taken into account. The overall sensitivity and specificity were 88.15 and $97.6 \%$ with a positive predictive value of $90.74 \%$ and a negative predictive value of $96.94 \%$ [21]. A limitation of this study included the retrospective design and the selection bias that occurs when preselecting only 2 confocal images for review. Preselecting a limited number of images highlighting key CSLM morphological features of selected lesions potentially increases the likelihood that observers would recognize the selected lesion correctly, and may reduce the distraction of viewing a larger number of images that include nondiagnostic features.

Gerger et al. [29] also conducted a diagnostic accuracy study with the CSLM involving a series of benign and malignant melanocytic and nonmelanocytic skin lesions. Four dermatooncologists received a 1-hour standardized instruction of the diagnostic features of nevi, melanoma, basal cell cancer and seborrheic keratoses. Two diagnostic images of 162 skin tumors were shown on a computer screen, and a diagnosis was rendered. Using classification and regression analyses, a 3-step algorithm based on 3 criteria led to a correct classification in $96.30 \%$ of melanoma, $98.89 \%$ of benign nevi and $100 \%$ of basal cell carcinomas and seborrheic keratoses [29].

Pellacani et al. [30] have also evaluated a series of benign and malignant melanocytic lesions to determine the diagnostic significance of certain morphological features of CSLM. In this study, they examined 102 melanocytic lesions including 49 nevi, 16 Spitz/Reed nevi and 37 melanomas. The lesions were examined to determine the frequency of morphological features seen in benign and malignant melanocytic lesions and their diagnostic significance for melanoma identification. In multivariate analyses, 6 criteria were most strongly correlated with a diagnosis of melanoma: presence of nonedged dermal papillae; atypical cells; isolated nucleated cells within dermal papillae; pagetoid cells; widespread pagetoid infiltration, and cerebriform clusters. These investigators designed a diagnostic algorithm using these morphological criteria, to enable the correct classification of all melanomas in a retrospective analysis [30].

Diagnostic Accuracy of CSLM Compared to Dermoscopy in Melanocytic Lesions
These authors have very recently evaluated the sensitivity and specificity of confocal features for the diagnosis of 351 equivocal melanocytic lesions including 136 melanomas and 215 nevi [31]. Two blinded observers evaluated these lesions for 37 morphological features and performed statistical analysis $\left(\chi^{2}\right.$, multivariate discriminant analysis and binary logistic regression) to identify the most significant confocal criteria and to test the model proposed above. Six morphological criteria that were independently correlated with melanoma by discriminant analysis included: roundish pagetoid cells; nonedged papillae; epidermal disarray; nucleated cells in the dermal papillae, and pleomorphic pagetoid infiltration. The presence of junctional nests was a negative feature for malignancy. A CSLM score was also calculated for each lesion in which 2 major features (nonedged papillae and cellular atypia at the dermal-epidermal junction) and 4 minor criteria (roundish pagetoid cells, widespread pagetoid infiltration, cerebriform nests and nucleated cells within the papillae) as proposed in the previous algorithm by Pellacani et al. [30] using a proposed threshold of 3 resulted in a sensitivity of $91.9 \%$ and a specificity of $69.3 \%$ [31].

We report the first prospective diagnostic accuracy study to compare dermoscopy and CSLM performed in real time, at the bedside, involving 125 patients with clinically suspicious pigmented skin lesions. The specificities of CSLM and dermoscopy were essentially similar with results of 83.0 and $84.1 \%$, respectively. Improvements in the sensitivity of CSLM over dermoscopy were obtained with values of $97.3 \%$ with CSLM compared to $89.2 \%$ with dermoscopy, although the result was not statistically significant. Of particular interest is however the benefit of combining the two methods for improvement in diagnostic accuracy. In the present analysis, dermoscopy misclassified 4 melanomas whereas CSLM misclassified 1 melanoma. Of importance, the two techniques did not misclassify the same lesions and, as a result, when combining both tests no melanomas were misdiagnosed. These results suggest that these techniques may be complementary and there may be benefits in combining these modalities to improve the diagnostic accuracy of melanoma.

Two of the melanomas misclassified by dermoscopy in our study were amelanotic/hypomelanotic melanomas. In these cases, architectural and cytological features of melanoma were evident with CSLM facilitating the correct classification of the lesions. Amelanotic melanomas are a recognized diagnostic pitfall in the clinical and dermoscopic diagnosis of melanoma $[32,33]$.

Dermatology 2007;215:365-372 
Despite the importance of an early diagnosis of melanoma, amelanotic melanomas lack the conventional clinical and dermoscopic features of melanoma, and the diagnosis may be delayed with the potential of disease progression. Pizzichetta et al. [32] conducted a retrospective analysis of 151 amelanotic/hypomelanotic skin lesions which included 10 amelanotic melanomas to evaluate if dermoscopy was a useful technique for the diagnosis of amelanotic/hypomelanotic melanoma. Peripheral pigmentation was not helpful in the diagnosis of amelanotic/hypomelanotic melanoma, as this feature was also identified in hypopigmented benign melanocytic lesions. Vascular patterns (linear irregular vessels or the combination of dotted and linear irregular vessels) were the only dermoscopic criteria reliably seen in the 'truly' amelanotic melanomas and were felt to be useful in distinguishing amelanotic/hypomelanotic melanoma permitting a correct diagnosis in 4 of 10 cases. The presence of vascular patterns with a peripheral pigment network has been identified as being of diagnostic importance for melanoma [32-38], and in pure amelanotic melanomas milky red globules and/or areas with irregular linear vessels may be useful [38]. Validation of these dermoscopic criteria in amelanotic melanoma is pending, given the rarity of these lesions and the lack of large-scale prospective studies. Skvara et al. [39] conducted a retrospective study of 325 melanocytic lesions missed by dermoscopy and analyzed these lesions with pattern analysis, the $\mathrm{ABCD}$ rule of dermoscopy and the 7-point checklist. No dermoscopic features or pattern of features were identified that could reliably discriminate between melanoma and nevi, suggesting that the value of dermoscopy is limited for early and featureless melanomas, and the follow-up of such lesions is important. In such cases, sequential dermoscopic imaging has been shown to improve the detection of featureless melanomas [40]. Busam et al. [15] examined 2 patients with amelanotic melanoma using CSLM. Architectural and cytological features of melanoma were identified permitting in vivo detection of clinically amelanotic melanoma. Taken together, CSLM provides useful additional information in these cases that are not provided with conventional clinical and dermoscopic examination. The use of CSLM in this setting may facilitate the improved diagnostic accuracy of melanoma, particularly when used in combination with dermoscopy.

Our study demonstrates that CSLM is a reliable noninvasive instrument that facilitates the in vivo recognition of cutaneous melanoma. A major strength of our study is that this analysis was done in a prospective manner and the analysis involved a comprehensive and systematic review of 30,089 images with the diagnosis being rendered prospectively and preoperatively. All imaging was done by the same confocal observer (R.L.), and a diagnosis was made during the imaging session. As a result, the confocal observer remained blinded to the final diagnosis. The assessment of patients clinically and with the dermatoscope prior to CSLM imaging provides insight into how diagnostic accuracy and clinical judgement of patients with pigmented lesions might be improved by integration of noninvasive imaging techniques at the bedside.

However, the diagnostic accuracy we report with CSLM differs from previous studies. Gerger et al. [21] report relatively higher specificity values and positive predictive values than our study of 97.6 and $90.74 \%$ compared to 82.1 and $69.4 \%$. These changes may reflect the study design (retrospective vs. prospective) and the number of images reviewed. We reviewed on average $241 \mathrm{im}$ ages per patient compared to 2 preselected images in Gerger et al. [21].

Our study has certain limitations. First, it is a singlecenter trial and the investigator who examined the patients with dermoscopy and CSLM has extensive experience in both, and as a result it is not possible to generalize these results. The accuracy we report with dermoscopy is however similar to recent research and metaanalysis reported to date. In a series of 198 melanocytic lesions, Annessi et al. [24] report sensitivity, specificity and diagnostic accuracy of melanoma using the pattern analysis method of $85.4,74.5$ and $82.3 \%$, respectively. In addition, a recent metaanalysis of 13 dermoscopic studies indicated similar results with a diagnostic accuracy of dermoscopy of $83.2 \%$ and a specificity of $85.8 \%$ [25]. Secondly, dermoscopy was performed initially by the same investigator, followed by CSLM. Areas of atypia identified by dermoscopy guided the initial placement of the CSLM on the lesion and could have influenced the diagnosis rendered, and the tests may not have been independent. This approach was however deliberate as we sought to evaluate the utility of this instrument in the clinic in a realistic setting. We believe that if CSLM is utilized at the bedside, dermoscopy will be used initially, and CSLM analysis should follow.

In conclusion, our research suggests that CSLM holds potential as a useful noninvasive technology in the assessment of benign and malignant skin lesions. CSLM had a relatively higher sensitivity over dermoscopy with similar specificity. No melanomas were misidentified 
when both techniques were combined, suggesting that CSLM and dermoscopy are complementary. Our research adds to the growing literature that supports the ongoing investigation of this technique and the conduct of a large multicenter trial.

\section{Acknowledgement}

We acknowledge the work of Kara Thompson, MSc, and Adam Webber, MSc, Biostatisticians for this project. This project was funded by grants from the Canadian Dermatology Foundation, Nova Scotia Health Research Foundation and the University Internal Medicine Research Foundation.

\section{References}

1 Kopf AW, Mintzis M, Bart RS: Diagnostic accuracy in malignant melanoma. Arch Dermatol 1975;111:1291-1292.

$\checkmark 2$ Curley RK, Cook MG, Fallowfield ME, Marsden RA: Accuracy in clinically evaluating pigmented lesions. BMJ 1989;299:16-18.

3 Wolf IH, Smolle J, Soyer HP, Kerl H: Sensitivity in the clinical diagnosis of malignant melanoma. Melanoma Res 1998;8:425-429.

4 Pehamberger H, Steiner A, Wolff K: In vivo epiluminescence microscopy of pigmented skin lesions. I. Pattern analysis of pigmented skin lesions. J Am Acad Dermatol 1987;17: 571-583.

5 Steiner A, Wolff K, Pehamberger H: In vivo epiluminescence microscopy of pigmented skin lesions. II. Diagnosis of small pigmented skin lesions and early detection of malignant melanoma. J Am Acad Dermatol 1987; 17:584-591.

-6 Argenziano G, Soyer HP, Chimenti S, Talamini R, Corona R, Sera F, et al: Dermoscopy of pigmented lesions: results of a consensus meeting via the Internet. J Am Acad Dermatol 2003;48:679-693.

-7 Binder M, Schwarz M, Winkler A, Steiner A, Kaider A, Wolff K, et al: Epiluminescence microscopy: a useful tool for the diagnosis of pigmented skin lesions for formerly trained dermatologists. Arch Dermatol 1995;131: 286-291.

-8 Marghoob AA, Swindle LD, Moricz CZ, Sanchez Negron FA, Slue B, Halpern AC, et al: Instruments and new technologies for the in vivo diagnosis of melanoma. J Am Acad Dermatol 2003;49:777-797.

-9 Rajadhyaksha M, Grossman M, Esterowiz D, Webb RH, Anderson RR: In vivo confocal scanning laser microscopy of human skin: melanin provides strong contrast. J Invest Dermatol 1995; 104:946-952.

-10 Rajadhyaksha M, Gonzalez S, Zavislan JM, Anderson RR, Webb RH: In vivo confocal scanning laser microscopy of human skin. II. Advances in instrumentation and comparison with histology. J Invest Dermatol 1999; 113:293-303.

11 Langley RG, Rajadhyaksha M, Dwyer P, Anderson RR, Sober AJ: Confocal scanning laser microscopy of pigmented skin lesions. J Invest Dermatol 1996;106:836
12 Langley RG, Rajadhyaksha M, Dwyer PJ, Sober AJ, Flotte TJ, Anderson RR: Confocal scanning laser microscopy of benign and malignant melanocytic lesions in vivo. J Am Acad Dermatol 2001;45:365-376.

13 Selkin B, Rajadhyaksha M, Gonzalez S, Langley RG: In vivo confocal microscopy in dermatology. Dermatol Clin 2001;19:369377.

14 Busam KJ, Charles C, Lee G, Halpern AC: Morphologic features of melanocytes, pigmented keratinocytes and melanophages by in vivo confocal scanning laser microscopy. Mod Pathol 2001;14:862-868.

15 Busam KJ, Hester K, Charles C, Sachs DL, Antonescu CR, Gonzalez S, et al: Detection of clinically amelanotic malignant melanoma and assessment of its margins by in vivo confocal scanning laser microscopy. Arch Dermatol 2001;137:923-929.

16 Busam KJ, Charles C, Lohmann CM, Marghoob A, Goldgeier M, Halpern AC: Detection of intraepidermal malignant melanoma in vivo by confocal scanning laser microscopy. Melanoma Res 2002;12:349-355.

17 Tannous ZS, Mihm MC, Flotte TJ, Gonzalez $S$ : In vivo examination of lentigo maligna and malignant melanoma in situ, lentigo maligna type, by near-infrared reflection confocal microscopy: comparison of in vivo confocal images with histological sections. J Am Acad Dermatol 2002;46:260-263.

18 Pellacani G, Cesinaro AM, Grana C, Seidenari S: In vivo confocal scanning laser microscopy of pigmented Spitz nevi: comparison of in vivo confocal images with dermoscopy and routine histopathology. J Am Acad Dermatol 2004;51:371-376.

19 Pellacani G, Cesinaro AM, Longo C, Grana C, Seidenari S: Microscopic in vivo description of cellular architecture of dermoscopic pigment network in nevi and melanomas. Arch Dermatol 2005;141:147-154.

20 Pellacani G, Cesinaro AM, Seidenari S: Reflectance-mode confocal microscopy for the in vivo characterization of pagetoid melanocytosis in melanomas and nevi. J Invest Dermatol 2005; 125:532-537.

21 Gerger A, Koller S, Kern T, Massone C, Steiger K, Richtig E, et al: Diagnostic applicability of in vivo confocal laser scanning microscopy in melanocytic skin tumors. J Invest Dermatol 2005;124:493-498.
22 Langley RG, Burton E, Walsh N, Propperova I, Murray SJ: In vivo confocal scanning laser microscopy of benign lentigines: comparison to conventional histology and in vivo characteristics of lentigo maligna. J Am Acad Dermatol 2006;55:88-97.

23 Binder M, Puespoeck-Schwarz M, Steiner A, Kittler H, Muellner M, Wolff K, et al: Epiluminescence microscopy of small pigmented skin lesions: short-term formal training improves the diagnostic performance of dermatologists. J Am Acad Dermatol 1997;36: 197-202.

24 Annessi G, Bono R, Sampogna F, Faraggiana T, Abeni D: Sensitivity, specificity, and diagnostic accuracy of three dermoscopic algorithmic methods in the diagnosis of doubtful melanocytic lesions. J Am Acad Dermatol 2007;56:759-767.

- 25 Kittler H, Pehamberger H, Wolff K, Binder M: Diagnostic accuracy of dermoscopy. Lancet Oncol 2002;3:159-165.

26 Fisher LD, van Belle G: Biostatistics: A Methodology for the Health Sciences. New York, Wiley \& Sons, 1993.

27 Hawass N: Comparing the sensitivities and specificities of two diagnostic procedures on the same group of patients. Br J Radiol 1997; 70:360-366.

28 Scope A, Benvenuto-Andrade C, Agero AL, Malvehy J, Puig S, Rajadhyaksha M, et al: In vivo reflectance confocal microscopy imaging of melanocytic skin lesions: consensus terminology glossary and illustrative images. J Am Acad Dermatol 2007;57:644-658.

29 Gerger A, Koller S, Weger W, Richtig E, Kerl $\mathrm{H}$, Samonigg H, et al: Sensitivity and specificity of confocal laser-scanning microscopy for in vivo diagnosis of malignant skin tumors. Cancer 2006;107:193-200.

30 Pellacani G, Cesinaro AM, Seidenari S: Reflectance mode confocal microscopy of pigmented skin lesions - improvement in melanoma diagnostic specificity. J Am Acad Dermatol 2005;53:979-985.

31 Pellacani G, Guitera P, Longo C, Avramidis $\mathrm{M}$, Seidenari S, Menzies S: The impact of in vivo reflectance confocal microscopy for the diagnostic accuracy of melanoma and equivocal melanocytic lesions. J Invest Dermatol DOI: $10.1038 /$ si.jid.5700993. 
>32 Pizzichetta MA, Talamini R, Stanganelli I, Puddu P, Bono R, Argenziano G, et al: Amelanotic/hypomelanotic melanoma: clinical and dermoscopic features. Br J Dermatol 2004;150:1117-1124.

33 Zalaudek I, Argenziano G, Di Stefani A, Ferrara G, Marghoob AA, Hofmann-Wellenhof $\mathrm{R}$, et al: Dermoscopy in general dermatology. Dermatology 2006;212:7-18.

34 Stolz W, Braun-Falco O, Bilek P, Landthaler M: Color Atlas of Dermatoscopy, ed 2. Berlin, Blackwell, 2002, pp 42-57.
35 Zalaudek I, Argenziano G, Kerl H, Soyer HP, Hofmann-Wellenhof R: Amelanotic/hypomelanotic melanoma: is dermatoscopy useful for diagnosis? J Dtsch Dermatol Ges 2003;1:369-373.

36 Argenziano G, Zalaudek I, Corona R, Sera F, Cicale L, Petrillo G, et al: Vascular structures in skin tumors: a dermoscopy study. Arch Dermatol 2004;140:1485-1489.

37 de Giorgi V, Sestini S, Massi D, Maio V, Giannotti B: Dermoscopy for 'true' amelanotic melanoma: a clinical dermoscopicpathologic case study. J Am Acad Dermatol 2006;54:341-344.
38 Cavicchini S, Tourlaki A, Bottini S: Dermoscopic vascular patterns in nodular 'pure' amelanotic melanoma. Arch Dermatol 2007; 143:556.

>39 Skvara H, Teban L, Fiebiger M, Binder M, Kittler H: Limitations of dermoscopy in the recognition of melanoma. Arch Dermatol 2005;141:155-160.

40 Kittler H, Guitera P, Riedl E, Avramidis M, Teban L, Fiebiger M, et al: Identification of clinically featureless incipient melanoma using sequential dermoscopy imaging. Arch Dermatol 2006;142:1113-1119. 